\title{
Searching for Sentences Expressing Opinions by using Declaratively Subjective Clues
}

\author{
Nobuaki Hiroshima, Setsuo Yamada, Osamu Furuse and Ryoji Kataoka \\ NTT Cyber Solutions Laboratories, NTT Corporation \\ 1-1 Hikari-no-oka Yokosuka-Shi Kanagawa, 239-0847 Japan \\ hiroshima.nobuaki@lab.ntt.co.jp
}

\begin{abstract}
This paper presents a method for searching the web for sentences expressing opinions. To retrieve an appropriate number of opinions that users may want to read, declaratively subjective clues are used to judge whether a sentence expresses an opinion. We collected declaratively subjective clues in opinionexpressing sentences from Japanese web pages retrieved with opinion search queries. These clues were expanded with the semantic categories of the words in the sentences and were used as feature parameters in a Support Vector Machine to classify the sentences. Our experimental results using retrieved web pages on various topics showed that the opinion expressing sentences identified by the proposed method are congruent with sentences judged by humans to express opinions.
\end{abstract}

\section{Introduction}

Readers have an increasing number of opportunities to read opinions (personal ideas or beliefs), feelings (mental states), and sentiments (positive or negative judgments) that have been written or posted on web pages such as review sites, personal web sites, blogs, and BBSes. Such subjective information on the web can often be a useful basis for finding out what people think about a particular topic or making a decision.

A number of studies on automatically extracting and analyzing product reviews or reputations on the web have been conducted (Dave et al., 2003; Morinaga et al., 2002; Nasukawa and Yi, 2003; Tateishi et al., 2004; Kobayashi et al.,
2004). These studies focus on using sentiment analysis to extract positive or negative information about a particular product. Different kinds of subjective information, such as neutral opinions, requests, and judgments, which are not explicitly associated with positive/negative assessments, have not often been considered in previous work. Although sentiments provide useful information, opinion-expressing sentences like "In my opinion this product should be priced around \$15," which do not express explicitly positive or negative judgments (unlike sentiments) can also be informative for a user who wants to know others' opinions about a product. When a user wants to collect opinions about an event, project, or social phenomenon, requests and judgments can be useful as well as sentiments. With open-domain topics, sentences expressing sentiments should not be searched exclusively; other kinds of opinion expressing sentences should be searched as well.

The goal of our research is to achieve a web search engine that locates opinion-expressing sentences about open-domain topics on products, persons, events, projects, and social phenomena. Sentence-level subjectivity/objectivity classification in some of the previous research (Riloff and Wiebe, 2003; Wiebe and Riloff, 2005) can identify subjective statements that include speculation in addition to positive/negative evaluations. In these efforts, the subjectivity/objectivity of a current sentence is judged based on the existence of subjective/objective clues in both the sentence itself and the neighboring sentences. The subjective clues, some adjective, some noun, and some verb phrases, as well as other collocations, are learned from corpora (Wiebe, 2000; Wiebe et al., 2001). Some of the clues express subjective meaning unrestricted to positive/negative measurements. The sentence-level subjectivity ap- 
proach suggests a way of searching for opinion expressing sentences in the open domain.

The problem of applying sentence-level subjectivity classification to opinion-expressing sentence searches is the likelihood of collecting too many sentences for a user to read. According to the work of Wiebe et al. (2001), 70\% of sentences in opinion-expressing articles like editorials and $44 \%$ of sentences in non-opinion expressing articles like news reports were judged to be subjective. In analyzing opinions (Cardie et al., 2003; Wilson et al., 2004), judging document-level subjectivity (Pang et al., 2002; Turney, 2002), and answering opinion questions (Cardie et al., 2003; Yu and Hatzivassiloglou, 2003), the output of a sentence-level subjectivity classification can be used without modification. However, in searching opinion-expressing sentences, it is necessary to designate criteria for opinion-expressing sentences that limit the number of retrieved sentences so that a user can survey them without difficulty. While it is difficult to formally define an opinion, it is possible to practically tailor the definition of an opinion to the purpose of the application (Kim and Hovy, 2004).

This study introduces the notion of declaratively subjective clues as a criterion for judging whether a sentence expresses an opinion and proposes a method for finding opinionexpressing sentences that uses these clues. Declaratively subjective clues such as the subjective predicate part of the main clause and subjective sentential adverb phrases suggest that the writer is the source of the opinion. We hypothesize that a user of such an "opinion-expressing sentence" search wants to read the writer's opinions and that explicitly stated opinions are preferred over quoted or implicational opinions. We suppose that writer's ideas or beliefs are explicitly declared in a sentence with declaratively subjective clues whereas sentences without declaratively subjective clues mainly describe things. The number of sentences with declaratively subjective clues is estimated to be less than the number of subjective sentences defined in the previous work. We expect that the opinion expressing sentences identified with our method will be appropriate from the both qualitative and quantitative viewpoints.

Section 2 describes declaratively subjective clues and explains how we collected them from opinion-expressing sentences on Japanese web pages retrieved with opinion search queries. Section 3 explains our strategy for searching opin- ion-expressing sentences by using declaratively subjective clues. Section 4 evaluates the proposed method and shows how the opinionexpressing sentences found by the proposed method are congruent with the sentences judged by humans to be opinions.

\section{Declaratively Subjective Clues}

Declaratively subjective clues are a basic criterion for judging whether a sentence expresses an opinion. We extracted the declaratively subjective clues from Japanese sentences that evaluators judged to be opinions.

\subsection{Opinion-expressing Sentence Judgment}

We regard a sentence to be "opinion expressing" if it explicitly declares the writer's idea or belief at a sentence level. We define as a "declaratively subjective clue", the part of a sentence that contributes to explicitly conveying the writer's idea or belief in the opinion-expressing sentence. For example, "I am glad" in the sentence "I am glad to see you" can convey the writer's pleasure to a reader, so we regard the sentence as an "opinionexpressing sentence" and "I am glad" as a "declaratively subjective clue." Another example of a declaratively subjective clue is the exclamation mark in the sentence "We got a contract!" It conveys the writer's emotion about the event to a reader.

If a sentence only describes something abstract or concrete even though it has word-level or phrase-level subjective parts, we do not consider it to be opinion expressing. On the other hand, some word-level or phrase-level subjective parts can be declaratively subjective clues depending on where they occur in the sentence. Consider the following two sentences.

(1) This house is beautiful.

(2) We purchased a beautiful house.

Both (1) and (2) contain the word-level subjective part "beautiful". Our criterion would lead us to say that sentence (1) is an opinion, because "beautiful" is placed in the predicate part and (1) is considered to declare the writer's evaluation of the house to a reader. This is why "beautiful" in (1) is eligible as a declaratively subjective clue. On the other hand, sentence (2) is not judged to contain an opinion, because "beautiful" is placed in the noun phrase, i.e., the object of the verb "purchase," and (2) is considered to report the event of the house purchase rather ob- 
jectively to a reader. Sentence (2) partially contains subjective information about the beauty of the house; however this information is unlikely to be what a writer wants to emphasize. Thus, "beautiful" in (2) does not work as a declaratively subjective clue.

These two sentences illustrate the fact that the presence of a subjective word ("beautiful") does not unconditionally assure that the sentence expresses an opinion. Additionally, these examples do suggest that sentences containing an opinion can be judged depending on where such wordlevel or phrase-level subjective parts as evaluative adjectives are placed in the predicate part.

Some word-level or phrase-level subjective parts such as subjective sentential adverbs can be declaratively subjective clues depending on where they occur in the sentence. In sentence (3), "amazingly" expresses the writer's feeling about the event. Sentence (3) is judged to contain an opinion because there is a subjective sentential adverb in its main clause.

(3) Amazingly, few people came to my party.

The existence of some idiomatic collocations in the main clause also affects our judgment as to what constitutes an opinion-expressing sentence. For example, sentence (4) can be judged as expressing an opinion because it includes "my wish is”.

(4) My wish is to go abroad.

Thus, depending on the type of declaratively subjective clue, it is necessary to consider where the expression is placed in the sentence to judge whether the sentence is an opinion.

\subsection{Clue Expression Collection}

We collected declaratively subjective clues in opinion-expressing sentences from Japanese web pages. Figure 1 illustrates the flow of collection of eligible expressions.

\begin{tabular}{|l|l|}
\hline type & query’s topic \\
\hline \hline Product & cell phone, car, beer, cosmetic \\
\hline Entertainment & sports, movie, game, animation \\
\hline Facility & museum, zoo, hotel, shop \\
\hline Politics & diplomacy, election \\
\hline Phenomena & diction, social behavior \\
\hline Event & firework, festival \\
\hline Culture & artwork, book, music \\
\hline Organization & company \\
\hline Food & cuisine, noodle, ice cream \\
\hline Creature & bird \\
\hline
\end{tabular}

Table 1: Topic Examples

First, we retrieved Japanese web pages from forty queries covering a wide range of topics such as products, entertainment, facilities, and phenomena, as shown in Table 1 . We used queries on various topics because we wanted to acquire declaratively subjective clues for opendomain opinion web searches. Most of the queries contain proper nouns. These queries correspond to possible situations in which a user wants to retrieve opinions from web pages about a particular topic, such as "Cell phone $\mathrm{X}$," "Y museum," and "Football coach Z's ability", where $\mathrm{X}, \mathrm{Y}$, and $\mathrm{Z}$ are proper nouns.

Next, opinion-expressing sentences were extracted from the top twenty retrieved web pages in each query, 800 pages in total. There were 75,575 sentences in these pages.

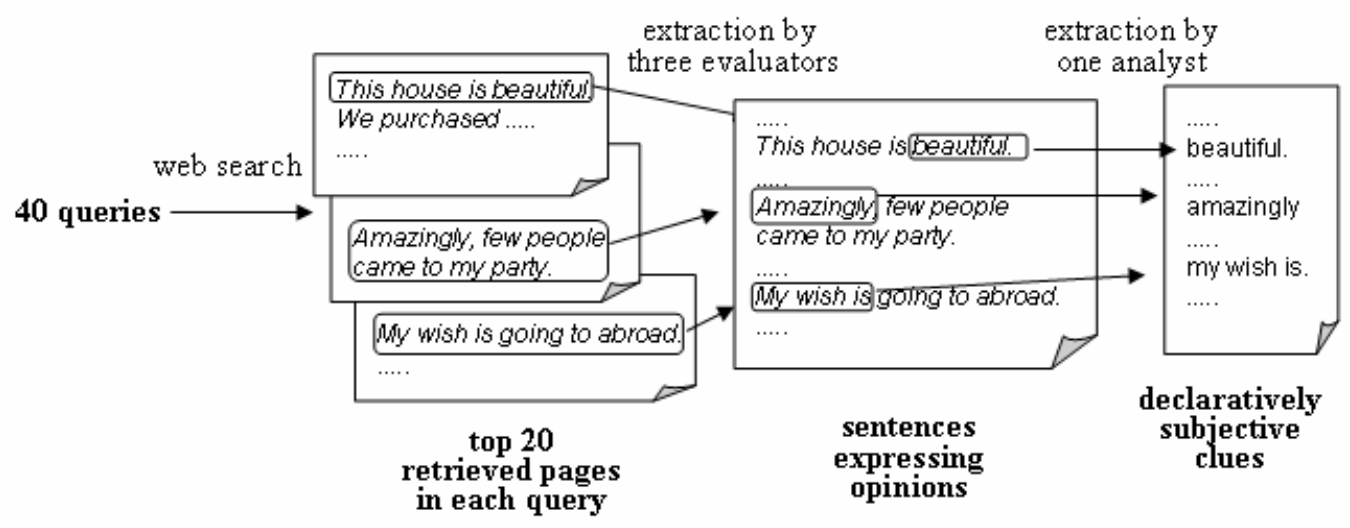

Figure 1: Flow of Clue Expression Collection 


\begin{tabular}{|c|c|c|}
\hline & type & $\begin{array}{c}\text { example sentence } \\
\text { (English translation of Japanese sentence) }\end{array}$ \\
\hline (a) & Thought & $\begin{array}{l}\text { Kono hon wa kare no dato omou. } \\
\text { (I think this book is his.) }\end{array}$ \\
\hline (b) & Declarative adverb & $\begin{array}{l}\text { Tabun rainen yooroppa ni iku. } \\
\text { (I will possibly go to Europe next year.) }\end{array}$ \\
\hline (c) & Interjection & $\begin{array}{l}\text { Waa, suteki. } \\
\text { (Oh, wonderful.) }\end{array}$ \\
\hline (d) & Intensifier & $\begin{array}{l}\text { Karera wa totemo jouzu ni asonda. } \\
\text { (They played extremely well) }\end{array}$ \\
\hline (e) & Impression & $\begin{array}{l}\text { Kono yougo wa yayakoshii. } \\
\text { (This terminology is confusing.) }\end{array}$ \\
\hline (f) & Emotion & $\begin{array}{l}\text { Oai dekite ureshii desu. } \\
\text { (I am glad to see you.) }\end{array}$ \\
\hline (g) & Positive/negative judgment & $\begin{array}{l}\text { Anata no oodio kiki wa sugoi. } \\
\text { (Your audio system is terrific.) }\end{array}$ \\
\hline (h) & Modality about propositional attitude & $\begin{array}{l}\text { Sono eiga wo miru beki da. } \\
\text { (You should go to the movie.) }\end{array}$ \\
\hline (i) & Value judgment & $\begin{array}{l}\text { Kono bun wa imi fumei da. } \\
\text { (This sentence makes_no sense.) }\end{array}$ \\
\hline (j) & Utterance-specific sentence form & $\begin{array}{l}\text { Towa ittemo,ima wa tada no yume dakedo. } \\
\text { (Though, it's literally just a dream now.) }\end{array}$ \\
\hline (k) & Symbol & $\begin{array}{l}\text { Keiyaku wo tottazo! } \\
\text { (We got a contract!) }\end{array}$ \\
\hline (l) & Idiomatic collocation & $\begin{array}{l}\text { Ii nikui. } \\
\text { (It's hard to say.) }\end{array}$ \\
\hline (m) & Uncertainty & $\begin{array}{l}\text { Ohiru ni nani wo tabeyou kanaa. } \\
\text { (I am wondering what I should eat for lunch.) }\end{array}$ \\
\hline (n) & Imperative & $\begin{array}{l}\text { Saizen wo tukushi nasai. } \\
\text { (Give it your best.) }\end{array}$ \\
\hline
\end{tabular}

Table 2: Clue Types

Three evaluators judged whether each sentence contained an opinion or not. The 13,363 sentences judged to do so by all three evaluators were very likely to be opinion expressing. The number of sentences which three evaluators agreed on as non-opinion expressing was $42,346 .{ }^{1}$ Out of the 13,363 opinion expressing sentences, 8,425 were then used to extract declaratively subjective clues and learn positive examples in a Support Vector Machine (SVM), and 4,938 were used to assess the performance of opinion expressing sentence search (Section 4). Out of the 42,346 non-opinion sentences, 26,340 were used to learn negative examples, and 16,006 were used to assess, keeping the number ratio of the positive and negative example sentences in learning and assessing.

One analyst extracted declaratively subjective clues from 8,425 of the 13,363 opinionexpressing sentences, and another analyst checked the result. The number of declaratively

\footnotetext{
${ }^{1}$ Note that not all of these opinion-expressing sentences retrieved were closely related to the query because some of the pages described miscellaneous topics.
}

subjective clues obtained was 2,936. These clues were classified into fourteen types as shown in Table 2, where the underlined expressions in example sentences are extracted as declaratively subjective clues. The example sentences in Table 2 are Japanese opinion-expressing sentences and their English translations. Although some English counterparts of Japanese clue expressions might not be cogent because of the characteristic difference between Japanese and English, the clue types are likely to be language-independent. We can see that various types of expressions compose opinion-expressing sentences.

As mentioned in Section 2.1, it is important to check where a declaratively subjective clue appears in the sentence in order to apply our criterion of whether the sentence is an opinion or not. The clues in the types other than (b), (c) and (l) usually appear in the predicate part of a main clause.

The declaratively subjective clues in Japanese examples are placed in the rear parts of sentences except in types (b), (c) and (l). This reflects the heuristic rule that Japanese predicate 
parts are in principle placed in the rear part of a sentence.

\section{Opinion-Sentence Extraction}

In this section, we explain the method of classifying each sentence by using declaratively subjective clues.

The simplest method for automatically judging whether a sentence is an opinion is a rule-based one that extracts sentences that include declaratively subjective clues. However, as mentioned in Section 2, the existence of declaratively subjective clues does not assure that the sentence expresses an opinion. It is a daunting task to write rules that describe how each declaratively subjective clue should appear in an opinionexpressing sentence. A more serious problem is that an insufficient collection of declaratively subjective clues will lead to poor extraction performance.

For that reason, we adopted a learning method that binarily classifies sentences by using declaratively subjective clues and their positions in sentences as feature parameters of an SVM. With this method, a consistent framework of classification can be maintained even if we add new declaratively subjective clues, and it is possible that we can extract the opinion-expressing sentences which have unknown declaratively subjective clues.

\subsection{Augmentation by Semantic Categories}

Before we can use declaratively subjective clues as feature parameters, we must address two issues:

- Cost of building a corpus: It is costly to provide a sufficient amount of tagged corpus of opinion-expressing-sentence labels to ensure that learning achieves a high-performance extraction capability.

- Coverage of words co-occurring with declaratively subjective clues: Many of the declaratively subjective clue expressions have co-occurring words in the opinion-expressing sentence. Consider the following two sentences.

(5) The sky is high.

(6) The quality of this product is high.

Both (5) and (6) contain the word "high" in the predicate part. Sentence (5) is considered to be less of an opinion than (6) because an evaluator might judge (5) to be the objective truth, while all evaluators are likely to judge (6) to be an opinion. The adjective "high" in the predicate part can be validated as a declaratively subjective clue depending on co-occurring words. However, it is not realistic to provide all possible co-occurring words with each declaratively subjective clue expression.

Semantic categories can be of help in dealing with the above two issues. Declaratively subjective clue expressions can be augmented by semantic categories of the words in the expressions. An augmentation involving both declaratively subjective clues and co-occurrences will increase feature parameters. In our implementation, we adopted the semantic categories proposed by Ikehara et al. (1997). Utilization of semantic categories has another effect: it improves the extraction performance. Consider the following two sentence patterns:

(7) $\mathrm{X}$ is beautiful.

(8) $\mathrm{X}$ is pretty.

The words "beautiful" and "pretty" are adjectives in the common semantic category, "appearance", and the degree of declarative subjectivity of these sentences is almost the same regardless of what $\mathrm{X}$ is. Therefore, even if "beautiful" is learned as a declaratively subjective clue but "pretty" is not, the semantic category "appearance" that the learned word "beautiful" belongs to, enables (8) to be judged opinion expressing as well as (7).

\subsection{Feature Parameters to Learn}

We implemented our opinion-sentence extraction method by using a Support Vector Machine (SVM) because an SVM can efficiently learn the model for classifying sentences into opinionexpressing and non-opinion expressing, based on the combinations of multiple feature parameters. The following are the crucial feature parameters of our method.

- $\quad 2,936$ declaratively subjective clues

- $\quad 2,715$ semantic categories that words in a sentence can fall into

If the sentence has a declaratively subjective clue of type (b), (c) or (l) in Table 2, the feature parameter about the clue is assigned a value of 1 ; if not, it is assigned 0 . If the sentence has declaratively subjective clues belonging to types 


\begin{tabular}{|c|c|c|c|c|c|c|c|}
\hline \multirow{2}{*}{ Method } & \multicolumn{3}{|c|}{ Opinion } & \multicolumn{3}{|c|}{ No opinion } & \multirow{2}{*}{ Accuracy } \\
\hline & Precision & Recall & F-measure & Precision & Recall & F-measure & \\
\hline $\begin{array}{l}\text { Occurrences of DS clues } \\
\text { (baseline 1) }\end{array}$ & $66.4 \%$ & $35.3 \%$ & $46.0 \%$ & $82.6 \%$ & $94.5 \%$ & $88.1 \%$ & $80.5 \%$ \\
\hline $\begin{array}{l}\text { Bag of words } \\
\text { (baseline 2) }\end{array}$ & $80.9 \%$ & $64.2 \%$ & $71.6 \%$ & $89.6 \%$ & $95.3 \%$ & $92.4 \%$ & $88.0 \%$ \\
\hline Proposed & $78.6 \%$ & $70.8 \%$ & $74.4 \%$ & $91.3 \%$ & $94.0 \%$ & $92.6 \%$ & $88.6 \%$ \\
\hline
\end{tabular}

Table 4: Results for comparison with baseline methods

\begin{tabular}{|c|c|c|}
\hline Answer & Opinion & No opinion \\
\hline Opinion & $\mathrm{a}$ & $\mathrm{b}$ \\
\hline No opinion & $\mathrm{c}$ & $\mathrm{d}$ \\
\hline
\end{tabular}

Table 3: Number of sentences in a test set

other than (b), (c) or (l) in the predicate part, the feature parameter about the clue is assigned 1 ; if not, it is assigned 0 .

The feature parameters for the semantic category are used to compensate for the insufficient amount of declaratively subjective clues provided and to consider co-occurring words with clue expressions in the opinion-expressing sentences, as mentioned in Section 3.1.

The following are additional feature parameters.

- 150 frequent words

- 13 parts of speech

Each feature parameter is assigned a value of 1 if the sentence has any of the frequent words or parts of speech. We added these feature parameters based on the hypotheses that some frequent words in Japanese have the function of changing the degree of declarative subjectivity, and that the existence of such parts of speech as adjectives and adverbs possibly influences the declarative subjectivity. The effectiveness of these additional feature parameters was confirmed in our preliminary experiment.

\section{Experiments}

We conducted three experiments to assess the validity of the proposed method: comparison with baseline methods, effectiveness of position information in SVM feature parameters, and effectiveness of SVM feature parameters such as declaratively subjective clues and semantic categories.

All experiments were performed using the Japanese sentences described in Section 2.1. We used 8,425 opinion expressing sentences, which were used to collect declaratively subjective clues as a training set, and used 4,938 opinionexpressing sentences as a test set. We also used 26,340 non-opinion sentences as a training set and used 16,006 non-opinion sentences as a test set. The test set was divided into ten equal subsets. The experiments were evaluated with the following measures following the variable scheme in Table 3:

$$
\begin{aligned}
& P_{o p}=\frac{a}{a+b} \quad R_{o p}=\frac{a}{a+c} \\
& F_{o p}=\frac{2 P_{o p} R_{o p}}{P_{o p}+R_{o p}} \\
& P_{n o_{-} o p}=\frac{d}{c+d} \quad R_{\text {no_op }}=\frac{d}{b+d} \\
& F_{n o_{-} o p}=\frac{2 P_{n o_{-} o p} R_{n o_{-} o p}}{P_{n o_{-} o p}+R_{n o_{-} o p}} \\
& A=\frac{a+d}{a+b+c+d}
\end{aligned}
$$

We evaluated ten subsets with the above measures and took the average of these results.

\subsection{Comparison with Baseline Methods}

We first performed an experiment comparing two baseline methods with our proposed method. We prepared a baseline method that regards a sentence as an opinion if it contains a number of declaratively subjective clues that exceeds a certain threshold. The best threshold was set through trial and error at five occurrences. We also prepared another baseline method that learns a model and classifies a sentence using only features about a bag of words.

The experimental results are shown in Table 4. It can be seen that our method performs better than the two baseline methods. Though the difference between our method's results and those of the bag-of-words method seems rather small, the superiority of the proposed method cannot be rejected at the significance level of 5\% in t-test. 


\begin{tabular}{|c|c|c|c|c|c|c|c|}
\hline \multirow{2}{*}{ Position } & \multicolumn{3}{|c|}{ Opinion } & \multicolumn{3}{c|}{ No opinion } & \multirow{2}{*}{ Accuracy } \\
\cline { 2 - 7 } & Precision & Recall & F-measure & Precision & Recall & F-measure & \\
\hline All words & $76.8 \%$ & $70.6 \%$ & $73.5 \%$ & $91.2 \%$ & $93.4 \%$ & $92.3 \%$ & $88.0 \%$ \\
\hline Last 10 words & $\mathbf{7 8 . 6 \%}$ & $\mathbf{7 0 . 8} \%$ & $\mathbf{7 4 . 4 \%}$ & $\mathbf{9 1 . 3 \%}$ & $\mathbf{9 4 . 0} \%$ & $\mathbf{9 2 . 6 \%}$ & $\mathbf{8 8 . 6 \%}$ \\
\hline
\end{tabular}

Table 5: Results for feature parameters with position information

\begin{tabular}{|c|c|c|c|c|c|c|c|c|}
\hline \multicolumn{2}{|c|}{ Feature sets } & \multicolumn{3}{|c|}{ Opinion } & \multicolumn{3}{c|}{ No opinion } & Accuracy \\
\hline $\begin{array}{c}\text { DS } \\
\text { clues }\end{array}$ & $\begin{array}{c}\text { Semantic } \\
\text { categories }\end{array}$ & Precision & Recall & $\begin{array}{c}\text { F- } \\
\text { measure }\end{array}$ & Precision & Recall & $\begin{array}{c}\text { F- } \\
\text { measure }\end{array}$ & \\
\hline & & $71.4 \%$ & $53.2 \%$ & $60.9 \%$ & $87.7 \%$ & $94.1 \%$ & $90.8 \%$ & $85.2 \%$ \\
\hline $\mathrm{Y}$ & & $\mathbf{7 9 . 9 \%}$ & $64.3 \%$ & $71.2 \%$ & $89.6 \%$ & $\mathbf{9 5 . 0 \%}$ & $92.2 \%$ & $87.8 \%$ \\
\hline & $\mathrm{Y}$ & $76.1 \%$ & $68.9 \%$ & $72.2 \%$ & $90.7 \%$ & $93.3 \%$ & $92.0 \%$ & $87.5 \%$ \\
\hline $\mathrm{Y}$ & $\mathrm{Y}$ & $78.6 \%$ & $\mathbf{7 0 . 8} \%$ & $\mathbf{7 4 . 4 \%}$ & $\mathbf{9 1 . 3 \%}$ & $94.0 \%$ & $\mathbf{9 2 . 6 \%}$ & $\mathbf{8 8 . 6 \%}$ \\
\hline
\end{tabular}

Table 6: Results for effect of feature parameters

\subsection{Feature Parameters with Position In- formation}

We inspected the effect of position information of 2,936 declaratively subjective clues based on the heuristic rule that a Japanese predicate part almost always appears in the last ten words in a sentence. Instead of more precisely identifying predicate position from parsing information, we employed this heuristic rule as a feature parameter in the SVM learner for practical reasons.

Table 5 lists the experimental results. "All words" indicates that all feature parameters are permitted at any position in the sentence. "Last 10 words" indicates that all feature parameters are permitted only if they occur within the last ten words in the sentence.

We can see that feature parameters with position information perform better than those without position information in all evaluations. This result confirms our claim that the position of the feature parameters is important for judging whether a sentence is an opinion or not.

However, the difference did not indicate superiority between the two results at the significance level of 5\%. In the "last 10 word" experiment, we restricted the position of 422 declaratively subjective clues like (b), (c) and (l) in Table 2, which appear in any position of a sentence, to the same conditions as with the other types of 2,514 declaratively subjective clues. The fact that the equal position restriction on all declaratively subjective clues slightly improved performance suggests there will be significant improvement in performance from assigning the individual position condition to each declaratively subjective clue.

\subsection{Effect of Feature Parameters}

The third experiment was designed to ascertain the effects of declaratively subjective clues and semantic categories. The declaratively subjective clues and semantic categories were employed as feature parameters for the SVM learner. The effect of each particular feature parameter can be seen by using it without the other feature parameter, because the feature parameters are independent of each other.

The experimental results are shown in Table 6 . The first row shows trials using only frequent words and parts of speech as feature parameters. " $\mathrm{Y}$ " in the first and second columns indicates exclusive use of declaratively subjective clues and semantic categories as the feature parameters, respectively. For instance, we can determine the effect of declaratively subjective clues by comparing the first row with the second row.

The results show the effects of declaratively subjective clues and semantic categories. The results of the first row show that the method using only frequent words and parts of speech as the feature parameters cannot precisely classify subjective sentences. Additionally, the last row of the results clearly shows that using both declaratively subjective clues and semantic categories as the feature parameters is the most effective. The difference between the last row of the results and the other rows cannot be rejected even at the significance level of 5\%. 


\section{Conclusion and Future Work}

We proposed a method of extracting sentences classified by an SVM as opinion-expressing that uses feature sets of declaratively subjective clues collected from opinion-expressing sentences in Japanese web pages and semantic categories of words obtained from a Japanese lexicon. The first experiment showed that our method performed better than baseline methods. The second experiment suggested that our method performed better when extraction of features was limited to the predicate part of a sentence rather than allowed anywhere in the sentence. The last experiment showed that using both declaratively subjective clues and semantic categories as feature parameters yielded better results than using either clues or categories exclusively.

Our future work will attempt to develop an open-domain opinion web search engine. To succeed, we first need to augment the proposed opinion-sentence extraction method by incorporating the query relevancy mechanism. Accordingly, a user will be able to retrieve opinionexpressing sentences relevant to the query. Second, we need to classify extracted sentences in terms of emotion, sentiment, requirement, and suggestion so that a user can retrieve relevant opinions on demand. Finally, we need to summarize the extracted sentences so that the user can quickly learn what the writer wanted to say.

\section{References}

Claire Cardie, Janyce Wiebe, Theresa Wilson, and Diane J. Litman. 2003. Combining Low-Level and Summary Representations of Opinions. for MultiPerspective Question Answering. Working Notes New Directions in Question Answering (AAAI Spring Symposium Series) .

Kushal Dave, Steve Lawrence, and David M. Pennock. 2003. Mining the peanut gallery: Opinion extraction and semantic classification of product reviews. Proceedings of the 12th International World Wide Web Conference, 519-528.

Satoru Ikehara, Masahiro Miyazaki, Akio Yokoo, Satoshi Shirai, Hiromi Nakaiwa, Kentaro Ogura, Yoshifumi Ooyama, and Yoshihiko Hayashi. 1997. Nihongo Goi Taikei - A Japanese Lexicon. Iwanami Shoten. 5 volumes. (In Japanese).

Soo-Min Kim and Eduard Hovy. 2004. Determining the Sentiment of Opinions. Proceedings of the. COLING04.

Nozomi Kobayashi, Kentaro Inui, Yuji Matsumoto, Kenji Tateishi, and Toshikazu Fukushima. 2004. Collecting Evaluative Expressions for Opinion Extraction. Proceedings of the First International Joint Con- ference on Natural Language Processing (IJCNLP04), 584-589.

Satoshi Morinaga, Kenji Yamanishi, and Kenji Tateishi. 2002. Mining Product Reputations on the Web. Proceedings of the eighth ACM SIGKDD International Conference on Knowledge Discovery and Data Mining (KDD 2002).

Bo Pang, Lillian Lee, and Shivakumar Vaithyanathan. 2002. Thumbs up? Sentiment Classification using Machine Learning Techniques. Proceedings of the Conference on Empirical Methods in Natural Language Processing (EMNLP-2002), 76-86

Tetsuya Nasukawa and Jeonghee Yi. 2003. Sentiment Analysis: Capturing Favorability Using Natural Language Processing. Proceedings of the 2nd International Conference on Knowledge Capture(K-CAP 2003).

Ellen Riloff and Janyce Wiebe. 2003. Learning Extraction Patterns for Subjective Expressions. Proceedings of the Conference on Empirical Methods in Natural Language Processing (EMNLP-03), 105-112.

Kenji Tateishi, Yoshihide Ishiguro, and Toshikazu Fukushima, 2004. A Reputation Search Engine that Collects People's Opinions by Information Extraction Technology, IPSJ Transactions Vol. 45 No.SIG07, 115-123.

Peter Turney. 2002. Thumbs Up or Thumbs Down? Semantic Orientation Applied to Unsupervised Classification of Reviews. Proceedings of the 40th Annual Meeting of the Association for Computational Linguistics (ACL-2002), 417-424.

Janyce Wiebe. 2000. Learning Subjective Adjectives from Corpora. Proceedings of the 17th National Conference on Artificial Intelligence (AAAI -2000).

Janyce Wiebe, Theresa Wilson, and Matthew Bell. 2001. Identifying Collocations for Recognizing Opinions. Proceedings of ACL/EACL 2001 Workshop on Collocation.

Janyce Wiebe and Ellen Riloff. 2005. Creating Subjective and Objective Sentence Classifiers from Unannotated Texts. Proceedings of Sixth International Conference on Intelligent Text Processing and Computational Linguistics (CICLing-2005), 486-497.

Theresa Wilson, Janyce Wiebe, and Rebecca Hwa, 2004. Just how mad are you? Finding strong and weak opinion clauses. Proceeding of the AAAI Spring Symposium on Exploring Attitude and Affect in Text: Theories and Applications.

Hong $\mathrm{Yu}$ and Vasileios Hatzivassiloglou. 2003. Towards Answering Opinion Questions: Separating Facts from Opinions and Identifying the Polarity of Opinion Sentences. Proceedings of the Conference on Empirical Methods in Natural Language Processing (EMNLP-2003). 